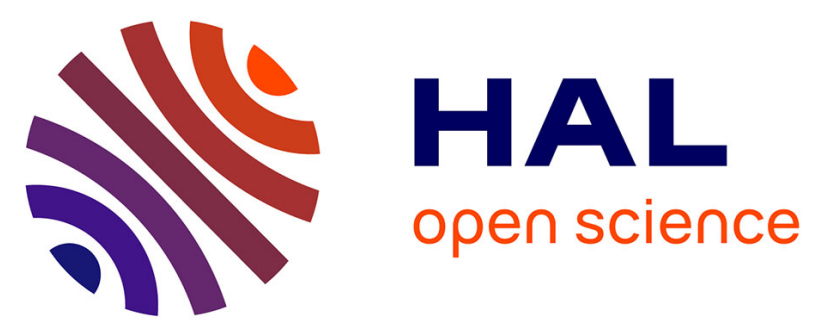

\title{
Study of the evolution of different dissipative solitons and phase measurement by Mach-Zehnder interferometry
}

\author{
V. Besse, C. Cassagne, Georges Boudebs, H. Leblond
}

\section{- To cite this version:}

V. Besse, C. Cassagne, Georges Boudebs, H. Leblond. Study of the evolution of different dissipative solitons and phase measurement by Mach-Zehnder interferometry. 2012 14th International Conference on Transparent Optical Networks (ICTON), Jul 2012, Coventry, United Kingdom. 10.1109/ICTON.2012.6253919 . hal-02442903

\section{HAL Id: hal-02442903 \\ https://hal.science/hal-02442903}

Submitted on 16 Apr 2021

HAL is a multi-disciplinary open access archive for the deposit and dissemination of scientific research documents, whether they are published or not. The documents may come from teaching and research institutions in France or abroad, or from public or private research centers.
L'archive ouverte pluridisciplinaire HAL, est destinée au dépôt et à la diffusion de documents scientifiques de niveau recherche, publiés ou non, émanant des établissements d'enseignement et de recherche français ou étrangers, des laboratoires publics ou privés. 


\title{
Study of the Evolution of Different Dissipative Solitons and Phase Measurement by Mach-Zehnder Interferometry
}

\author{
V. Besse, C. Cassagne, G. Boudebs ${ }^{(*)}$, H. Leblond \\ LUNAM Université, Université d'Angers, LPhiA, Laboratoire de Photoniques d'Angers \\ EA 4464, 49045 Angers Cedex 01, France \\ *Tel:(33) (0)2.41.73.54.26.Fax:(33)(0)2.41.73.52.16. email: georges.boudebs@univ-angers.fr
}

\begin{abstract}
We have studied numerically the evolution of soliton configurations according to the complex Ginzburg-Landau equation in presence of cubic-quintic non linearities in a 2-dimensional periodic potential. The aim was to establish a parameter "map" to predict experiments to be achieved using a Mach-Zehnder coupled to a "Spatial Light Modulator " into one arm for output phase measurements in order to fully characterize the soliton propagation.
\end{abstract}

Keywords: Soliton, cubic-quintic non linearities, Mach-Zehnder, Fourier transform, phase-shifting method.

\section{INTRODUCTION}

In conservative nonlinear systems, gain and loss are completely neglected and the dynamical equilibrium is reached due to the balance between nonlinear and dispersive (or diffractive) effects. However, the presence of gain and loss (due to optical amplifiers and saturable absorbers) in optically nonlinear media would influence the unique properties of localized structures in conservative (dissipativeless) nonlinear systems. Therefore, in order to form a dissipative optical soliton, there must also exist an additional balance between gain and losses [1,2].

\section{PRESENTATION OF THE CQ CGL EQUATION}

A generic model in the study of the pattern formation is based on the complex Ginzburg-Landau (CGL) equation with the cubic-quintic (CQ) (1) combination of nonlinear gain and loss terms, which are added to the linear. The CQ nonlinearity makes it possible to create solitary pulses, alias dissipative solitons [1], sitting on the stable zero background.

$$
\frac{\partial \mathrm{u}}{\partial \mathrm{Z}}=\left[-\delta+\mathrm{iV}(\mathrm{X}, \mathrm{Y})+\frac{i}{2} \nabla_{\perp}^{2}+(\mathrm{i}+\varepsilon)|\mathrm{u}|^{2}-(i v+\mu)|\mathrm{u}|^{4}\right] \mathrm{u}
$$

It describes the evolution of the amplitude of an electromagnetic field $u(X, Y, Z)$ in 2 dimensions $(X, Y)$ along the propagation direction $Z$. We denote by $\nabla_{\perp}{ }^{2}(2)$ the transverse Laplacian operator, i.e.:

$$
\nabla_{\perp}^{2}=\frac{\partial^{2}}{\partial X^{2}}+\frac{\partial^{2}}{\partial Y^{2}}
$$

The parameter $\delta$ is the linear loss, $\varepsilon$ is the cubic gain, $\mu$ is the quintic loss, $v$ is the quintic self-defocusing coefficient (saturation of the Kerr effect if $v>0)$. Here $\mathrm{V}(X, Y)(3)$ is a transverse periodic potential, with period $\pi$ and amplitude $\mathrm{V}_{0}$ :

$$
V(\mathrm{X}, \mathrm{Y})=V_{0}[\cos (2 \mathrm{X})+\sin (2 \mathrm{Y})]
$$

It is an obstacle to the mobility of the solitons, since it breaks the invariance of the CGL CQ equation through a Galilei transform. The parameter values chosen for simulations are $\delta=0.4, \varepsilon=1.85, \mu=1, v=0.1, V_{0}=-1$. This choice corresponds to a set of parameters for which the initial configuration is stable. We solved the CGL CQ (1) equation by means of a standard 4th-order Runge-Kutta scheme in the Z-direction, and five-point finite differences for the computation of the transverse Laplacian $\nabla_{\perp}{ }^{2}$. We used periodic boundary conditions. We give it an initial velocity to the soliton by multiplying the field $u_{0}$ of the steady state by a phase factor $\exp \left(\mathrm{i} \boldsymbol{k}_{0} \cdot \boldsymbol{X}\right)$. The key parameters are the norm $k_{0}$ of the initial wave vector $\boldsymbol{k}_{\boldsymbol{\theta}}(4)$, and the angle $\theta$ it makes with the X-axis. In other words, the initial transverse wave vector is:

$$
\mathrm{k}_{0}=\left(\mathrm{k}_{0} \cos \theta, \mathrm{k}_{0} \sin \theta, 0\right)
$$

In a laser setup, a small deviation of the propagation direction of the beam with respect to the Z-axis will produce such a phase factor. If $\mathrm{K}_{0}$ is the norm of the wave vector and $\varphi$ the angle of the deviation, the transverse wave vector has a norm $\mathrm{K}_{0} \sin \varphi$ in physical units, which corresponds to $k_{\theta}$ in normalized form.

We investigate the influence of the parameters $k_{0}$ and $\theta$ on the evolution of the solitons. 


\section{INFLUENCE OF THE CHOICE OF $\mathrm{k}_{\mathbf{0}}$ IN THE EVOLUTION OF THE NUMBER OF SOLITONS}

We studied the behaviour of the fundamental soliton [3](see Figure 1). We looked at the influence of parameters $k_{0}$ and $\theta$ on the evolution of the soliton pattern. For a small $\mathrm{k}_{-} 0$, the soliton merely oscillates in its site, while for the largest values it moves freely. In between, the motion of the soliton is accompanied by the creation of additional solitons, forming a line of stable solitons or 'arrow of solitons', while the initial soliton goes away. We studied the evolution of the number of solitons versus $k_{0}$ and $\theta$.
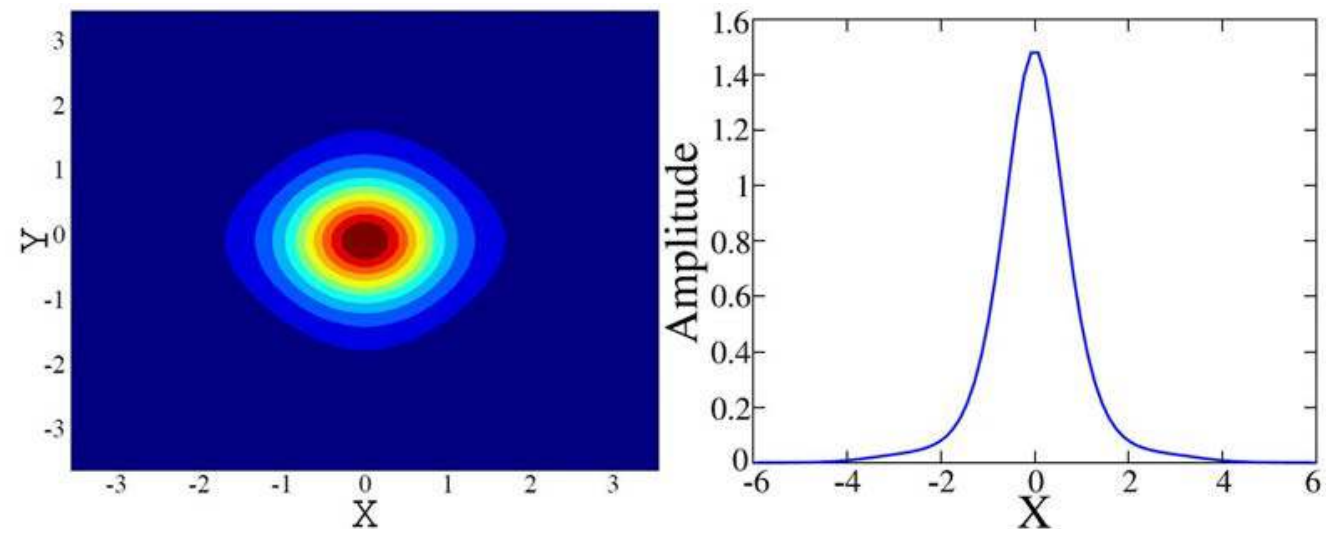

Figure 1. Fundamental soliton.

For $\theta=0$ we have plotted on Figure 2 the number of stable solitons, including the moving outgoing one, versus the initial kick $k_{0}$. Several domains can be seen. The number of solitons increases rapidly from 1 soliton for $\mathrm{k}_{0}=$ 1.6865 to a maximum of 6 solitons for $k_{0}=1.6927$ (see Figure 2). Beyond this value, the number of solitons decreases by steps of increasing length of the corresponding intervals of variation of the kick parameter. In the range $0 \leq \mathrm{k}_{0} \leq 1.6865$, the fundamental soliton only oscillates. For $1.6867 \leq \mathrm{k}_{0} \leq 2.081$, new solitons appear according to the following scenario:

i. $\quad$ some part (or all) of the soliton crosses the potential barrier

ii. it slows and lingers some time on the site

iii. then a part of the soliton crosses the next potential barrier

iv. the energy remaining in the site increases, and the spot stabilizes into a true fundamental soliton

These events recur until the soliton energy is no longer sufficient for it to cross the potential barrier or when it collides with another soliton. The maximum number of 6 solitons is reached for $1.6927 \leq \mathrm{k}_{0} \leq 1.6942$. For $k_{0}>2: 082$, the initial soliton moves across the simulation domain (see Figure 2).


Figure 2. Evolution of the number of outgoing solitons against $k_{0}$ and amplitude of fundamental soliton with $k_{0}=1.693$ and $\theta$ against $Z$.

\section{PHASE-SHIFTING METHODS (PSM) [4]}

Many actual research fields need to characterize phase, amplitude and shape of structure's mediums. Producing fringe pattern, several optical quantities can be extracted from the use of noninvasive interferometric techniques. Different methods exist as Twyman-Green interferometer [5], two-beam sharing interferometer [6], shear interferometer [7] and Mach-Zehnder interferometer [8] (MZI). Actually the automatic analysis of interferograms using digital image processing has become mature and specific applications in the domain of nonlinear photonics remain a subject of active research. 
The final goal of our study is to analyze the phase of a laser beam at the output of a non-linear medium where a nonlinear phase-shift is induced by high laser intensity profile. The Mach-Zehnder interferometer combined with a charge-coupled device (CCD) for image processing was proposed in [8]. With the previously proposed method described in details in [8] we could get intensity-resolved information by comparing the profile of the resolved phase, pixel per pixel, with that of the incident beam. So, it was possible to resolve the spatial profile of a complex nonlinear variation index using Fourier transform method with only one intense laser shot in the nonlinear material but the inconvenience was the poor spatial resolution especially when using focused beams. To improve this criterion, the phase-shifting interferometry technique is implemented here using a reflective Spatial Light Modulator (or SLM) instead of one mirror. The reliability of the proposed method is checked by direct comparison with a calibrated phase object already used for non linear characterizations(see details in [9])

a)

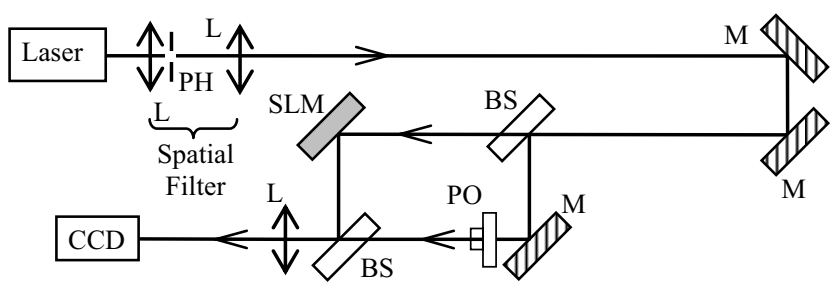

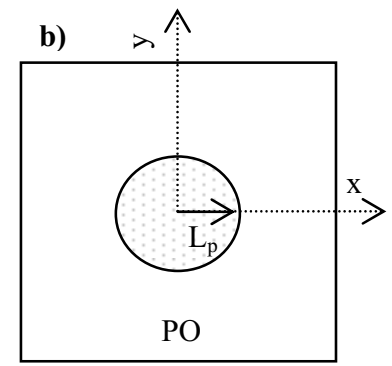

Figure 3: a) Experimental setup. PO: phase object; L: lenses; M: mirrors; BS: beam splitters; PH pin hole; SLM: spatial light modulator; b) Schematic of the PO, the circular phase object.

The built MZI is illuminated with an He-Ne laser emitting at $632.8 \mathrm{~nm}$ (see Fig. 3a). The first two lenses and the pinhole at the middle play the role of spatial filter to clean the beam. Then the collimated beam is divided into a test beam passing through the phase object (PO) to analyze and a reference beam where the mirror is replaced by a reflective Spatial Light Modulator (SLM). The SLM is based on a reflective liquid crystal on silicon microdisplays with $8 \mu \mathrm{m}$ pitch, $1920 \times 1080$ pixels resolution and is optimized to give a phase-shift above $2 \pi$ in the visible range with a maximum of $3.7 \pi$ at $\lambda=632 \mathrm{~nm}$. It is controlled by a software interface allowing the user to select a matrix level gray easy to transform via a graphic card into a matrix phase-shift. A $1388 \times 1038$ pixels CCD camera $(6.45 \mu \mathrm{m}$ pixel size) is placed at the MZI output. Generally, the final lens is placed at two times its focal length to form an image of the PO at the CCD.

The phase-changing circular plate consist of a glass structure on which a transparent dielectric disc (of radius $\mathrm{L}_{\mathrm{p}}=0.5 \mathrm{~mm}$ ) has been deposited (Fig. 3b). The disc has a thickness and index of refraction such that it retards the phase of the incident light by $\pi$ radians relative to the phase outside the disc at $1064 \mathrm{~nm}$. The PO serves as a test object, it was previously used in a $4 \mathrm{f}$ system for nonlinear refractive index measurement [9]. Theoretically, in our experiment (red HeNe laser) the phase-shift should be around: $632 \pi / 1064 \simeq 1.8$ rad.

By omitting the time dependency, we can generally express the nth interferogram acquired by the CCD: $I_{n}(x, y)=a(x, y)+b(x, y) \cos \left[\Delta \varphi(x, y)+\varphi_{n}\right]$, where $(x, y)$ is an arbitrary point in the interference pattern, $n=1,2, \ldots, N$ is an integer, $\varphi_{n}$ is a phase-shift given by the SLM and $\Delta \varphi(x, y)$ is the phase profile to be determined. From this simple expression, which contains three unknown parameters $a(x, y)$ (the background), $b(x, y)$ (the visibility) and $\Delta \varphi(x, y)$ one need to have at least three equations to find a solution to the problem. Available computing power nowadays allows real time numerical processing of a dozen interferograms using least-squares error criterion [10]. By the mean of a conventional trigonometric identity, $I_{n}$ can be rewritten: $I_{n}=a_{0}+a_{1} \cos \left(\varphi_{n}\right)+a_{2} \sin \left(\varphi_{n}\right)$. The unknowns are now $a_{0}=a, a_{1}=b \cos (\Delta \varphi)$ and $a_{2}=b \sin (\Delta \varphi)$. The interferograms must minimize the squared difference between $\mathrm{I}_{\mathrm{n}}$ and the acquisition giving a solution to standard matrix equation from which the required phase profile is calculated : $\Delta \varphi=\arctan \left(a_{2} / a_{1}\right)$ (for details see [4]). At this level of processing two dimensional unwrapping step is needed to remove the $2 \pi$ phase jumps. The image of the phase object obtained after numerically processing the $\mathrm{N}$ fringe patterns (here $\mathrm{N}=5$ ) is given in Fig. 4a. The phase when unwrapped shows a quasi-linear variation going from -5 (lower left corner) to 15 rad (upper right corner) where the $\mathrm{PO}$ is added on. $\Delta \varphi$, the phase distribution is the difference between the original unwrapped phase (shown in Fig. 4a) and a fitted based-plan phase (not shown here). In Fig. 4b we can see the result of PO, the local phase distribution, after subtraction. Finally, in Fig. 4c the central profile of the previous 
result is plotted along the $\mathrm{x}$ direction. Note that the mean value of the retrieved $\Delta \varphi$ is equal to the expected value $(1.8 \mathrm{rad})$ thus validating our numerical procedure.

a)

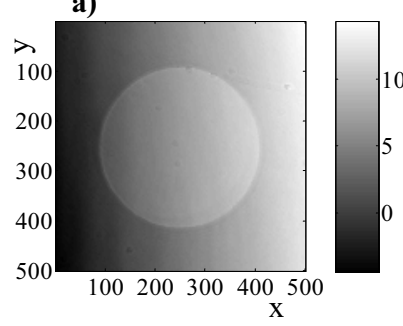

b)

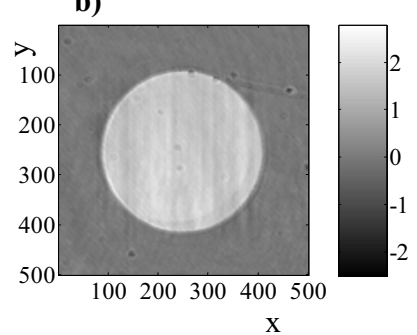

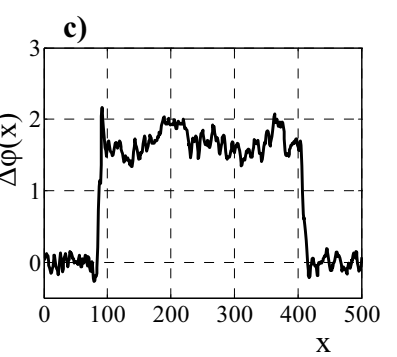

Figure 4 (a) Image of the PO obtained after numerical processing of the fringe patterns; (b) image of the PO obtained by the difference between phase values obtained in (a) and the linear fitting of the remaining phase after taking out a rectangular window containing the circular PO; the vertical bars indicate the phase in radians; (c) central profile of the phase distribution $\triangle \varphi$ in $P O$.

\section{CONCLUSIONS}

We have studied numerically the evolution of solitons configuration according to the complex Ginzburg-Landau equation in presence of cubic-quintic non linearities in a 2-dimensional periodic potential. The aim was to establish a parameter "map" to predict experiments for output phase measurements in order to fully characterize the soliton propagation. To improve the spatial resolution for input focused beam in nonlinear medium, the phase-shifting interferometery technique using the least-square error criterion was implemented with a reflective SLM inside a Mach-Zehnder configuration. The next step consists to implement this technique with pulsed laser in the picosecond range and to extract the phase at the output of thick non linear media according to the numerical result obtained in Section 3.

\section{REFERENCES}

[1] N. Akhmediev and A. Ankiewicz (Eds.), Dissipative Solitons, Lect. Notes Phys. 661, Springer, Berlin, 2005; N. Akhmediev and A. Ankiewicz (Eds.), Dissipative Solitons: From Optics to Biology and Medicine, Lect. Notes Phys. 751, Springer,Berlin, 2008.

[2] N. N. Rosanov, Spatial Hysteresis and Optical Patterns (Springer, Berlin, 2002); S. Barland et al., Nature (London) 419, 699 (2002); Z. Bakonyi, D. Michaelis, U. Peschel, G. Onishchukov, and F. Lederer, J. Opt. Soc. Am. B 19, 487 (2002);

[3] H. Leblond, B. A. Malomed, and D. Mihalache, Stable vortex solitons in the Ginzburg-Landau model of a two-dimensional lasing medium with a transverse grating, Phys. Rev. A 80, 033835 (2009).

[4] V. Besse, C. Cassagne, G. Boudebs, Spatially resolved phase objects using Mach-Zehnder interferometry, accepted to be published in Journal of Modern Optics, (2012).

[5] Y.-L. Chen, H.-C. Hsieh, W.-T. Wu, W.-Y. Chang, and D.-C. Su, Alternative method for measuring the full-field refractive index of a gradient-index lens with normal incidence heterodyne interferometry, Appl. Opt., 49, 6888-6892 (2010).

[6] S. De Nicola, P. Ferraro, A. Finizio, and G. Pierattini, Two-beam interferometer for measuring aberrations of optical components with axial symmetry, Appl. Opt., 40, 1631-1636 (2001).

[7] C. Falldorf, Measuring the complex amplitude of wave fields by means of shear interferometry, J. Opt. Soc. Am. A, 28, 1636-1647 (2011).

[8] G. Boudebs, M. Chis, X. Nguyen Phu, Third-order susceptibility measurement by a new Mach-Zehnder interferometry technique, J. Opt. Soc. Am. B, 18, 623-627 (2001).

[9] G. Boudebs and S. Cherukulappurath, Nonlinear optical measurements using a $4 f$ coherent imaging system with phase objects, Physical Review A, 69, 053813 (2004)

[10] J. E. Greivenkamp, Generalized data reduction for heterodyne interferometry, Opt. Eng., 23, 350-352 (1984). 